\title{
Identification and validation of p50 as the cellular target of eriocalyxin B
}

\author{
Ling-Mei Kong ${ }^{1,2, \ddagger}$, Xu Deng ${ }^{1, *}$, Zhi-Li Zuo ${ }^{1}$, Han-Dong Sun ${ }^{1}$, Qin-Shi Zhao ${ }^{1, *}$, Yan Li $^{1, *}$ \\ ${ }^{1}$ State Key Laboratory of Phytochemistry and Plant Resources in West China, Kunming Institute of Botany, Chinese Academy \\ of Sciences, Kunming 650201, China \\ ${ }^{2}$ Graduate University of the Chinese Academy of Sciences, Beijing 100039, China \\ ¥These authors contributed equally to this work \\ * Correspondence to: \\ Yan Li, email: liyanb@mail.kib.ac.cn \\ Qin-Shi Zhao, email: qinshizhao@mail.kib.ac.cn
}

Keywords: EriB, activity-based probes, p50, NF-kB signaling, apoptosis

Received: June 24, 2014

Accepted: September 12, 2014

Published: October 24, 2014

\section{ABSTRACT}

As an ent-kaurene diterpenoid isolated from Isodon eriocalyx var. Laxiflora, Eriocalyxin B (EriB) possesses potent bioactivity of antitumor and anti-autoimmune inflammation, which has been suggested to work through inhibition of NF-kappaB (NF-KB) signaling. However, the direct target of EriB remains elusive. In this study, we showed that EriB induced apoptosis is associated with the inhibition of NF-KB signaling in SMMC-7721 hepatocellular carcinoma cells. With activity-based probe profiling, we identified p50 protein as the direct target of EriB. We showed that cysteine 62 is the critical residue of $\mathrm{p} 50$ for EriB binding through the $\mathrm{a}, \boldsymbol{\beta}$-unsaturated ketones. As the result, EriB selectively blocks the binding between p50 and the response elements, whereas having no effect on the dimerization or the nuclear translocation of p50 and p65. SiRNA mediated knockdown of p50 attenuated the apoptosis induced by EriB in SMMC-7721 cells. Taken together, our studies illustrated that EriB induces cancer cell apoptosis through interfering with the binding between NF-KB and the response elements by targeting the cysteine 62 of p50, which highlights its potential for the development of p50 targeted cancer therapeutic agents.

\section{INTRODUCTION}

Isodon species (Labiatae) are widely distributed plants, many of which are used for the treatment of cancer and inflammation in Chinese folk medicine. Over the past twenty years, the structures and bioactivities of their diterpenoids constituents, especially those with an ent-kaurane skeleton, have received considerable phytochemical and biological attention [1]. Eriocalyxin B (EriB), an ent-kaurene diterpenoid (structure designated in Figure 1a) isolated from Isodon eriocalyx var. laxiflora, has been shown potent anti-tumor activity both in vitro and in vivo, and may be a promising candidate as an antitumor agent. A multitude of studies have been undertaken to elucidate the underlying mechanisms of the anti-tumor activity of EriB. NF- $\mathrm{KB}$, MAPK/ERK, AKT and $\mathrm{P} 53$ pathways have been reported, among which the NF- $\mathrm{KB}$ signaling is largely involved [2-7]. NF- $\mathrm{\kappa B}$ signaling regulates inflammation, tumorigenesis, cancer development, metastasis $[8]$ and chemoresistance $[9,10]$. The five members of NF- $\mathrm{kB}$ family, known as RelA (p65), RelB, c-Rel, p50/p105 (NF-kB1), and p52/p100 (NF-kB2), are sequestered in the cytoplasm as either homodimers or heterodimers. Upon activation, the inhibitory subunit I $\mathrm{B} \alpha$ is phosphorylated, ubiquitinated and degraded, which promotes the translocation of the NF- $\mathrm{kB}$ complex into the nucleus and activates the expression of the target genes $[11,12]$. It was proposed that EriB-mediated apoptosis of acute myeloid leukemia cells and the ovarian cancer stem cells was associated with NF-KB inactivation by preventing the I $\mathrm{\kappa} \mathrm{B} \alpha$ degradation and the subsequent nuclear translocation of NF- $\mathrm{KB}[2,3]$. Interfering with the binding of NF- $\mathrm{KB}$ subunits to the DNA response element without changing the translocation of NF- $\mathrm{kB}$ or their dimerization was reported to be responsible for cell apoptosis induced by EriB as well $[4,5]$. EriB inhibition of NF- $\mathrm{KB}$ pathway also contributed to the attenuation of autoimmune inflammation [6]. 
Though extensive investigations of the mechanism involved in the inhibition of NF- $\mathrm{KB}$ signaling by EriB have been reported, despite of some controversies, the direct targets of EriB remain unknown. To address the problem, we resorted to the technology of activity-based protein profiling (ABPP) strategy for solutions [13]. A series of labeled EriB probes were designed and synthesized by conjugating fluorophore or biotin with EriB. With the labeled activity-based probes, we investigated the direct target of EriB and the detailed mechanisms involved in its anti-tumor activity. Our data showed that EriB covalently modifies cysteine 62 of p50 through the $\alpha, \beta$-unsaturated ketones and EriB induces cancer cell apoptosis through interfering with the binding between NF- $\kappa \mathrm{B}$ and the response elements by directly targeting the cysteine 62 of $\mathrm{p} 50$.

\section{RESULTS}

\section{EriB inhibits NF-кB signaling and triggers cell apoptosis}

To begin with, the effect of EriB on the NF- $\kappa B$ activity was investigated with reporter activity assay. We observed that EriB significantly inhibited TNF- $\alpha$-induced activity of NF- $\kappa \mathrm{B}$ reporter (p65-Luc) in a concentrationdependent manner (Figure 1b). Further, we examined the effect of EriB on the expression of the endogenous target genes of NF- $\kappa \mathrm{B}$ signaling. Western blot analysis showed that EriB suppressed the expression of RelA [14] and XIAP [15] in SMMC-7721 cells. The anti-apoptotic proteins Bcl-2 and Bcl-xL, also known the downstream target genes of NF- $\mathrm{KB}$ [16], were down-regulated as

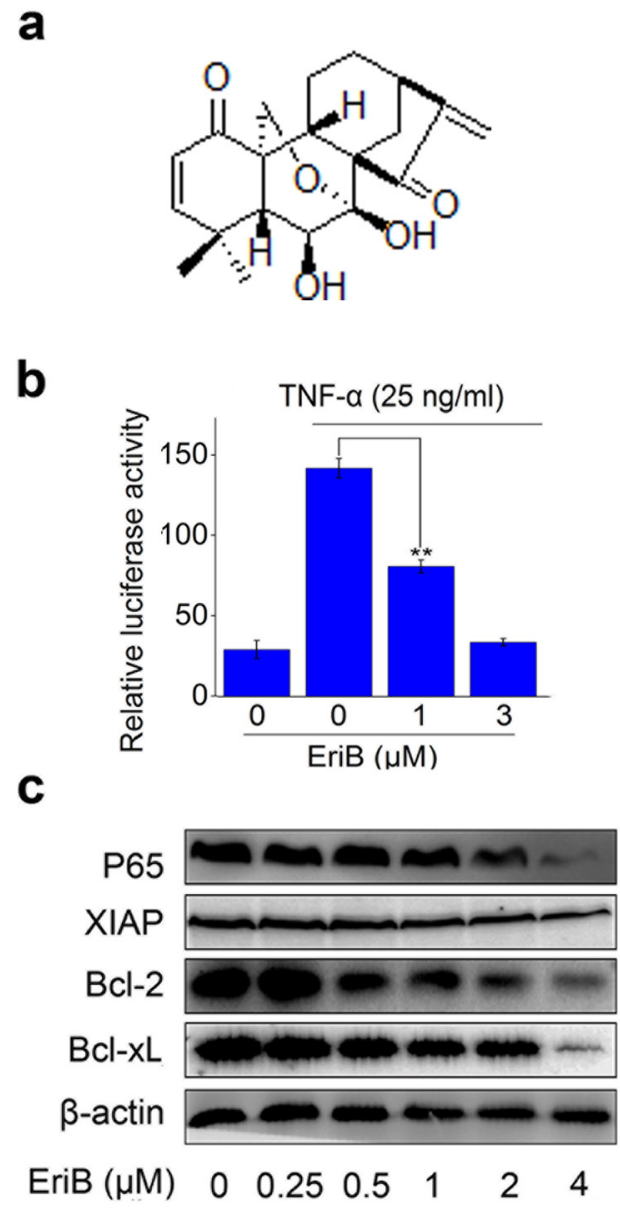

d

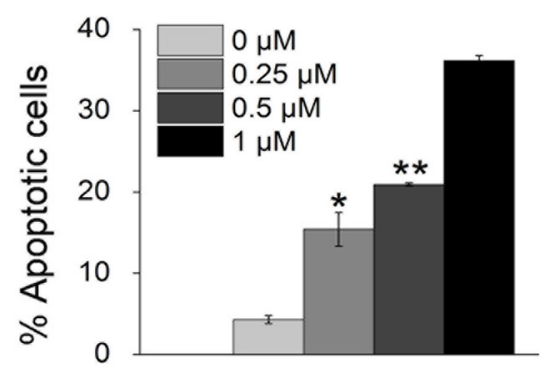

e

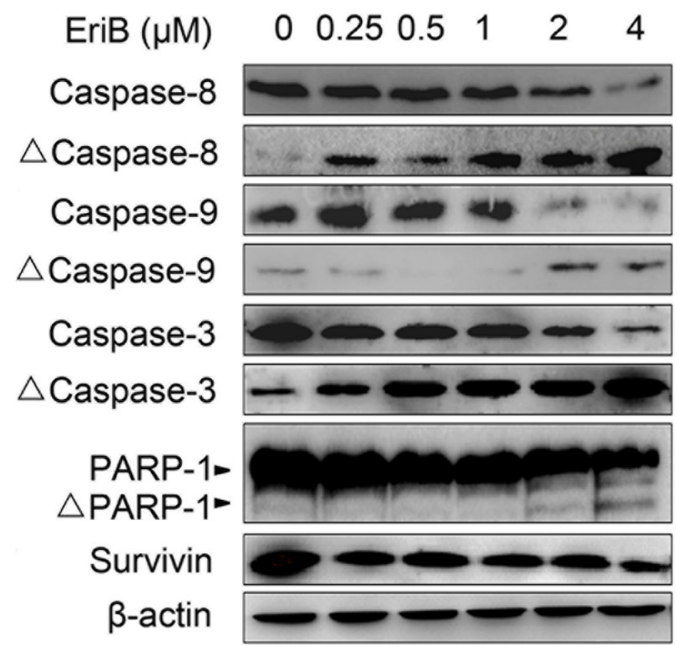

Figure 1: EriB inhibits NF-кB signaling and triggers apoptosis. (A) Structure of EriB. (B) HEK293T cells transiently transfeted with p65-Luc were pretreated with EriB and followed by activation with TNF- $\alpha$. The reporter activity was determined. The values represent the mean \pm S.D. $(n=3)$. (C) Western blot analysis of NF- $\kappa B$ downstream target proteins in SMMC-7721 cells with $\beta$-actin used as the loading control. (D) SMMC-7721 cells were treated with EriB for $48 \mathrm{~h}$. Apoptosis was analyzed by Annexin V-FITC/PI staining. The values represent the mean \pm S.D. ( $\mathrm{n}=3$ ). (E) Lysates from SMMC-7721 cells treated with EriB for $24 \mathrm{~h}$ were subjected to western blot analysis. $\beta$-actin was used as the loading control. Statistical significance was analyzed by One-way ANOVA, ${ }^{*} \mathrm{p}<0.01,{ }^{*} \mathrm{p}<0.05$. 
well by EriB when the concentration is over $0.5 \mu \mathrm{M}$ (Figure 1c). Taken together, EriB inhibited both intrinsic and TNF- $\alpha$-induced NF- $\kappa$ B activation.

Then the effect of EriB on cell apoptosis was checked by Annexin V-FITC/PI double staining. The result showed that EriB induced obvious apoptosis in SMMC7721 cells with a dose-dependent manner (Figure 1d), whereas no evident cell cycle arrest was observed in EriBtreated SMMC-7721 cells (Supplementary Information, Figure S1). There are two main pathways involved in apoptosis: one is the extrinsic pathway related to the activation of caspase-8, and the other is the intrinsic pathway regulated by $\mathrm{Bcl}-2$ family proteins and activation of caspase-9, which converge at caspase- 3 and follow by the activation of executive caspases [17, 18] and PARP-1 [19]. With western blotting assay, our results demonstrated that the activation of both caspase- 8 and caspase-9, as well as the cleavage of caspase-3 and PARP-1, were increased in EriB treated SMMC-7721 cells, whereas the expression of survivin, an inhibitor of caspase-3, 7 and 9 [20], was down-regulated (Figure 1e). Our findings showed that EriB induces tumor cell apoptosis as is associated with inhibited NF- $\kappa \mathrm{B}$ signaling, which is consistent with previous studies [2-6].

\section{Design and synthesis of activity-based probes of EriB}

Though extensive investigations of the mechanism involved in the inhibition of NF- $\mathrm{B}$ signaling by EriB have been reported, despite of some controversies, the direct targets of EriB remain unknown. To identify the cellular target of EriB and further elucidate the molecular mechanisms underlying the anti-tumor effects of EriB, the activity-based probes (ABPs) were designed and synthesized (Figure 2) (Supplementary Information, Figure S2). Typically, the ABP contains three components: the substrate that binds with the target protein, the hydrophilic spacer and the reporter, which is either a fluorophore for direct and sensitive detection, or a biotin to facilitate the isolation and enrichment of target proteins in complex proteomes. On the basis of the structureactivity relationship (SAR) studies of EriB [21], we know that the substitutions at C-6 hydroxyl group exert minimal implication on the activity. What's more, the C-6 hydroxyl group is highly chemically accessible. So the C-6 hydroxyl group was chosen as the site for the attachment. With polyethylene glycol as the hydrophilic linker, we installed a dansyl tag, the fluorophore commonly used in biological studies [22], to the C6 hydroxyl group to yield dansyl EriB (EBF6). Biotinyl EriB (EBB8) was directly accessed by condensation of biotin with EriB. EBF7, dansyl EriB with reduced $\alpha, \beta$-unsaturated ketones, was generated by catalytic hydrogenation of EBF6 with $\mathrm{Pd} / \mathrm{C}$ under $\mathrm{H}_{2}$, whose stereochemistry was characterized by NOESY. The cytotoxicity of the synthesized probes was determined, with the parent compound EriB used as the control (Figure 2). Both EBF6 and EBB8 exhibited comparable cytotoxicity toward SMMC-7721 cells to that of EriB. As expected, EBF7 lost the cytotoxicity due to the destroyed $\alpha, \beta$-unsaturated ketones, which were proved to be the key pharmacophores for the anti-tumor activity of EriB through structure-activity relationship studies [21].

\section{EriB directly binds to p50}

Next we extended our studies to elucidate the cellular target of EriB using the synthetic ABPs. It was observed that EBF6 distributed in both cytoplasm and nucleus of treated SMMC-7721 cells (Figure 3a).
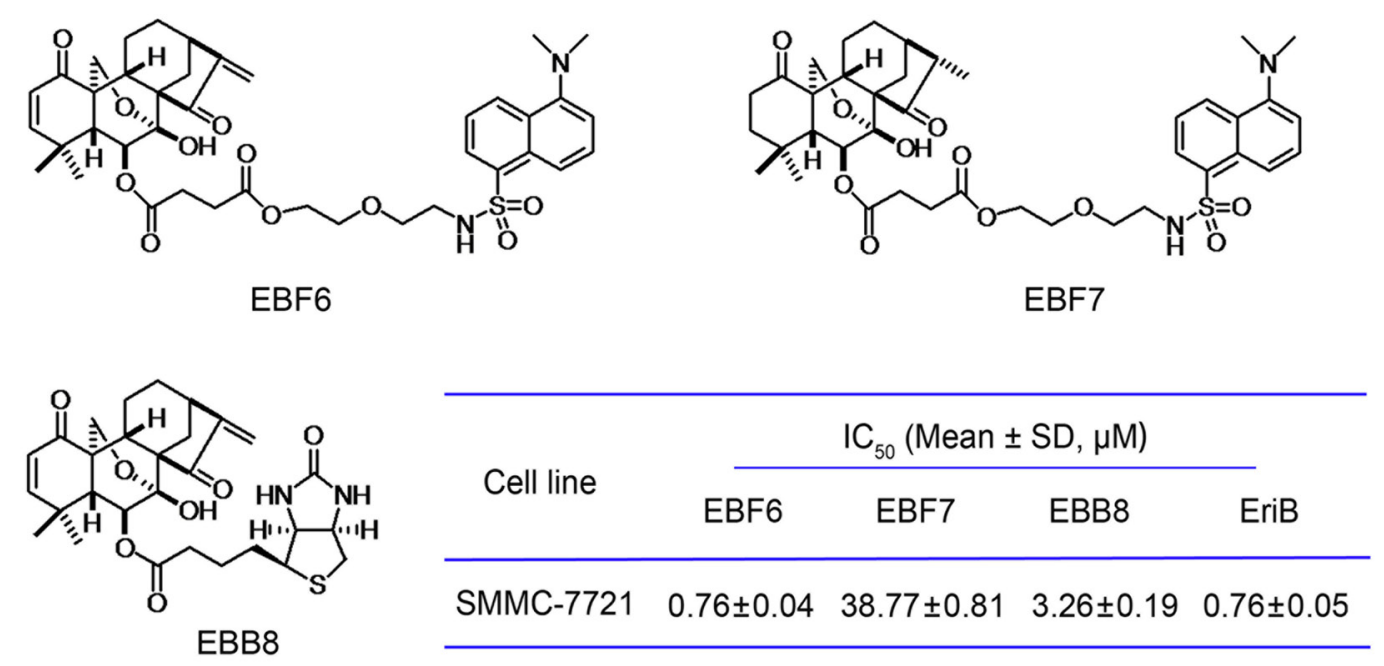

Figure 2: Chemical structures of EBF6, 7, EBB8 and their cytotoxicity against SMMC-7721 cells. 
To directly visualize the proteins labeled by EriB, cytoplasmic and nucleic lysates of SMMC-7721 cells were fractionated and incubated with EBF6 (1 $\mu \mathrm{M})$, respectively, followed by SDS-PAGE. The EBF6 bound proteins were detected with fluorescence under a UV trans-illuminator (UVP). As shown in Figure 3b, the cytoplasm and nucleus were successfully separated with the different expression of $\beta$-actin and Lamin $\mathrm{A} / \mathrm{C}$ as the demonstration and a specific protein band with molecular weight of $50 \mathrm{kDa}$ was detected both in cytoplasmic and nucleic fractions (lane 4 and 9), whereas no any band was detected under the treatment of either EriB or dansyl tag alone (DT5, Supplementary Information) (lane 2, 3 and $7,8)$. Moreover, the fluorescence of the labeled $50-\mathrm{kDa}$ protein band was competed off in the presence of a 3-fold excess of EriB both in cytoplasm and nucleus (Figure 3b, lane 5 and 10), indicating EriB binds with the 50-kDa protein specifically. When the cell lysates were treated with high concentration of $\operatorname{EBF} 6(5 \mu \mathrm{M})$, a few proteins were labeled and visualized, among which the $50-\mathrm{kDa}$ band appeared to be the predominant one (Supplementary Information, Figure S3).

As one crucial regulator of $\mathrm{NF}-\kappa \mathrm{B}$ signaling, $\mathrm{p} 50$, a protein of $50 \mathrm{kDa}$, dominantly dimerizes with $\mathrm{p} 65$ and is sequestered in the cytoplasm with the inhibitory subunit I $\kappa \mathrm{B}$ proteins in resting cells. Activation of NF- $\kappa \mathrm{B}$ signaling pathway leads to the release and translocation of p50/p65 dimer to the nucleus, and then activates the transcription of the target genes [23, 24]. So we postulated that the protein weighing $50 \mathrm{kDa}$ labeled by EriB was p50.

To verify our speculation, the biotinylated EriB (EBB8) was generated and incubated with SMMC-7721 cell lysates to directly pull down the potential proteins bound to EriB, with the streptavidin-coated beads as the immobilization strategy. As shown in Figure 3c, a $50-\mathrm{kDa}$ protein was captured by EBB8. When the membrane was subjected to immuno-blotting, the $50-\mathrm{kDa}$ band was recognized specifically by p50 antibody. Moreover, the interaction between EBB8 and p50 could be effectively competed by the co-presence of 10 -fold excess of EriB, which was consistent with the results observed in the competition experiment performed with EBF6, the fluorophore probe of EriB. The interaction of EriB with p50 was also observed in HEK293T cells. In HEK293T cells, treatment of TNF- $\alpha$ caused a decrease of p105 and an increase of p50 as reciprocation [25]. At the meanwhile the augment of p50 captured by EBB 8 was detected in TNF- $\alpha$ stimulated cells and EriB competed the interaction between EBB8 and p50 (Supplementary Information, Figure S4).

To further confirm the binding between EriB and p50, purified human recombinant GST-p50 was incubated with EBF6, with GST protein as a control. The result showed that EBF6 selectively interacted with p50 rather than the GST protein (Figure 3d).
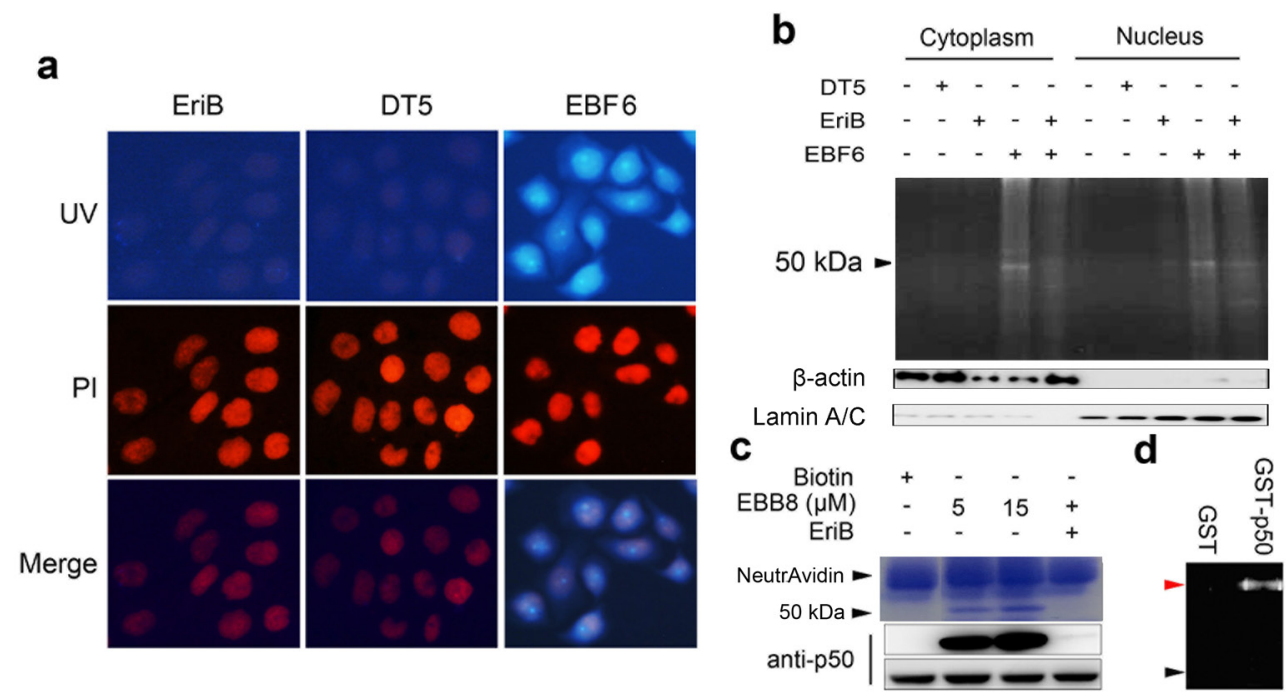

Figure 3: EriB selectively interacts with p50. (A) SMMC-7721 cells were incubated with $1 \mu$ M EriB, DT5 (dansyl tag alone, Supporting Information) and EBF6 for $3 \mathrm{~h}$, respectively, and the fluorescence was observed under microscopy. Nucleus and EBF6 were recognized by the red and blue fluorescence respectively. (B) The cytoplasmic fraction and nucleic fraction of SMMC-7721 cell lysates were incubated with EBF6 $(1 \mu \mathrm{M})$, with or without the presence of EriB, followed by resolving with SDS-PAGE and imaging under UV transilluminator. $\beta$-actin and Lamin $\mathrm{A} / \mathrm{C}$ are the loading controls of cytoplasmic fraction and the nucleic fraction respectively. (C) SMMC7721 cell lysates were incubated with EBB8 with or without the presence of EriB, or with biotin alone, followed by pull-down with streptavidin beads. The precipitates were resolved by SDS-PAGE, and the gel was stained with coomassie brilliant blue or subjected to immuno-blotting with p50 antibody, with p50 as the input. (D) Purified human recombinant GST and GST-p50 were incubated with EBF6 $(1 \mu \mathrm{M})$, respectively, for $3 \mathrm{~h}$ and after SDS-PAGE the samples were exposed to UV light. The red arrow indicates the GST-p50, and black arrow denotes GST. 


\section{EriB interferes with the binding of p50 to the response elements}

The hallmark of the activation of NF- $\mathrm{kB}$ signaling is the translocation of $\mathrm{p} 50 / \mathrm{p} 65$ dimer from the cytoplasm to the nucleus, and then binding to the response elements to activate the transcription. We explored the effects of the binding of EriB with p50 on these events. We first determined whether EriB interfered with the association of p50 and p65 in SMMC-7721 cells. Co-immunoprecipitation assay showed that TNF- $\alpha$ treatment increased the dimerization of p50 with p65, while pretreatment of EriB exerted little effects on the amount of p65-associated p50 (Figure 4a).

Next we examined the nuclear translocation of NF$\kappa \mathrm{B}$ by immunofluorescence staining of $\mathrm{p} 65$ protein. As shown in Figure $4 b$, obvious translocation of NF- $\kappa B$ was observed 30 min after TNF- $\alpha$ treatment. Pre-incubation of the cells with EriB did not block the translocation of NF- $\kappa$ B stimulated by TNF- $\alpha$, indicating that EriB does not affect the nuclear translocation of NF- $\mathrm{kB}$ either.

Subsequently, the effect of EriB on the interaction of $\mathrm{NF}-\kappa \mathrm{B}$ with the DNA response elements was investigated with chromatin immuno-precipitation assay. The results demonstrated that TNF- $\alpha$ markedly promoted the binding activity of NF- $\mathrm{KB}$ with the promoter of Bcl-xL as previously reported [26], whereas, which was effectively blocked by EriB dose-dependently (Figure 4c). Taken together, EriB, through direct interacting with p50, interfered with the DNA binding activity of $\mathrm{p} 50$ without affecting the dimerization or translocation of p50/ p65.

\section{EriB covalently modifies Cys62 of p50 through the $\alpha, \beta$-unsaturated ketones}

Structure-activity relationship studies of EriB revealed that the $\alpha, \beta$-unsaturated ketones were the key pharmacophores for the anti-tumor activity of EriB as previous reported [21]. To elucidate the structural basis of the binding of EriB with p50, in our present study, EBF7, dansyl EriB with $\alpha, \beta$-unsaturated ketones reduced was created, which, as expected, lost the anti-tumor activity in vitro ( $\mathrm{IC}_{50}>35 \mu \mathrm{M}$ in MTT assay) (Figure 2). Investigations were performed to verify the binding between p50 and EBF7. As expected, no fluorescence was observed in SMMC-7721 cells upon the treatment of EBF7 (Figure 5a). Consistently, the 50-kDa protein band on the gel was abolished as well from both the EBF7 treated cytoplasmic and the nuclear fractions (Figure 5b). These results indicated that the loss of binding capacity of EriB resulted from the reduction of $\alpha, \beta$-unsaturated ketones and thus $\alpha, \beta$-unsaturated ketones are crucial motifs for EriB to interact with p50.

$\alpha, \beta$-unsaturated ketones are known as Michael acceptors that potentially capture well-positioned nucleophiles at its target binding site, and may react with sulfhydryl groups of cysteine residues of proteins [27, 28]. Human p50 has seven cysteine residues at positions $62,88,119,124,162,262$, and $273[29,30]$. It is known that residues 59-71 (RYVCEGPSHGGLP) of p50 constitute the DNA binding domain of NF- $\mathrm{KB}$ [31-34], among which the sulphydryl group of Cys62 is an important determinant of DNA recognition by p50 subunit of NF-kB [35]. a

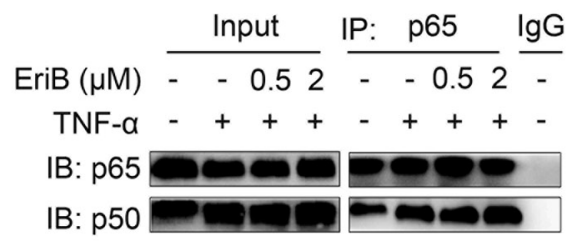

C

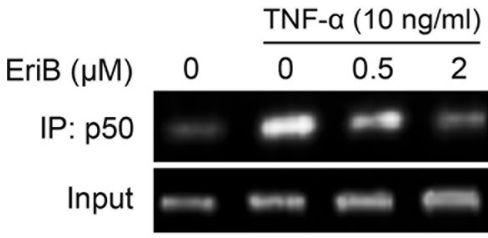

b

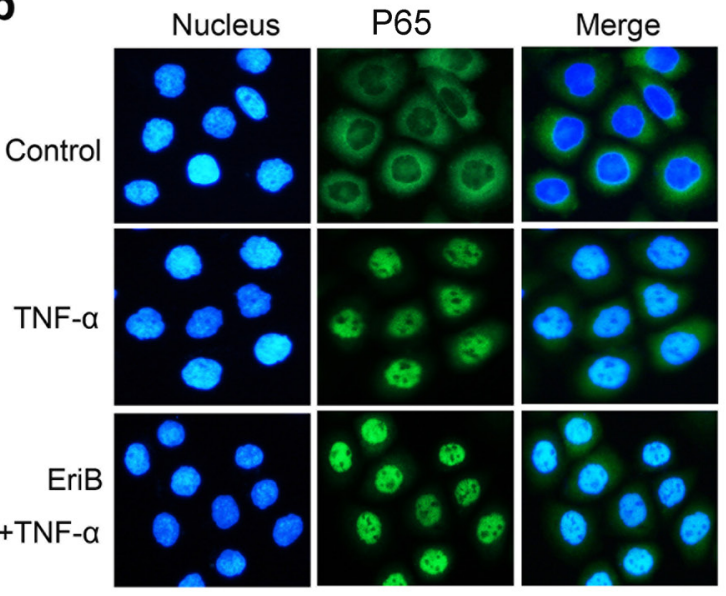

Figure 4: EriB interferes with the binding of p50 to the response elements. (A) SMMC-7721 cells were preincubated with EriB for $3 \mathrm{~h}$ before TNF- $\alpha(10 \mathrm{ng} / \mathrm{ml})$ stimulation. Immunoprecipitation was performed with anti-p65 antibody. P65 and the associated p50 were detected by immunoblotting. P65 and p50 were used as the inputs. (B) SMMC-7721 cells were pretreated with EriB (0.5 $\mu \mathrm{M})$ before $\mathrm{TNF}-\alpha(10 \mathrm{ng} / \mathrm{ml})$ stimulation. Distribution of NF- $\kappa \mathrm{B}$ was examined by immunofluorescence staining. NF- $\kappa \mathrm{B}$ and nucleus were recognized by the green and blue fluorescence, respectively. (C) SMMC-7721 cells were incubated with EriB at the indicated concentrations for $3 \mathrm{~h}$ before TNF- $\alpha$ stimulation. Chromatin immunoprecipitation assay was performed using p50 antibody. NF- $\kappa \mathrm{B}$-associated DNA was analyzed by PCR with the histone 3-associated RPL30 DNA as the input. 
a

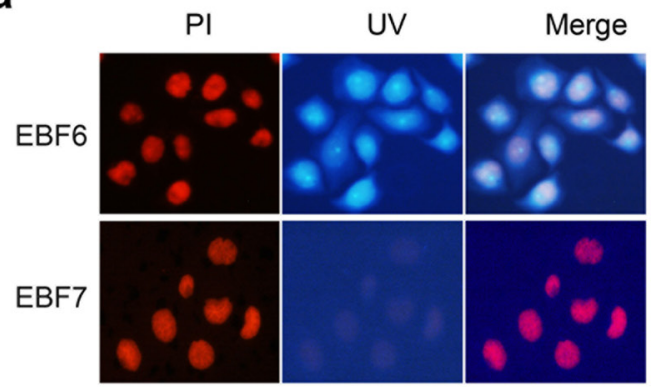

C

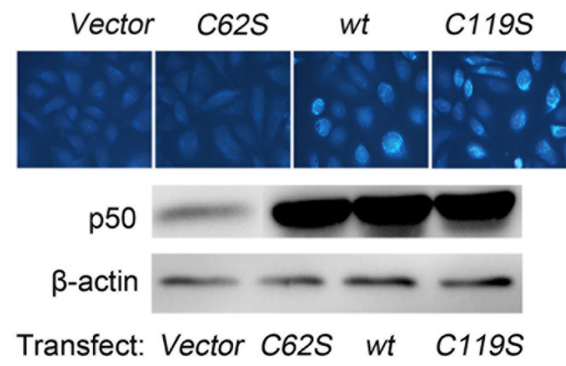

b

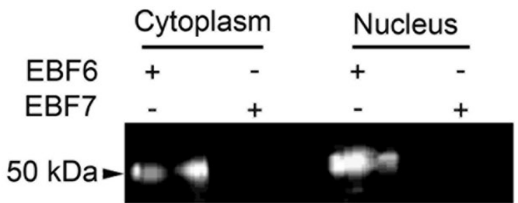

d

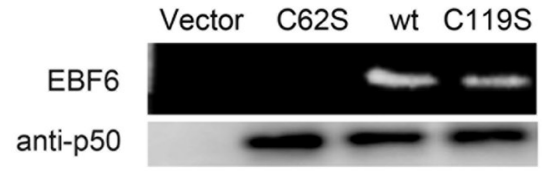

f

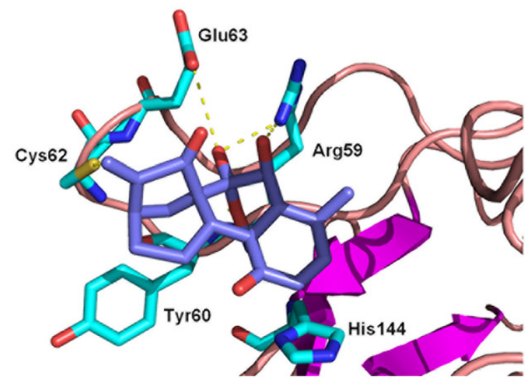

e

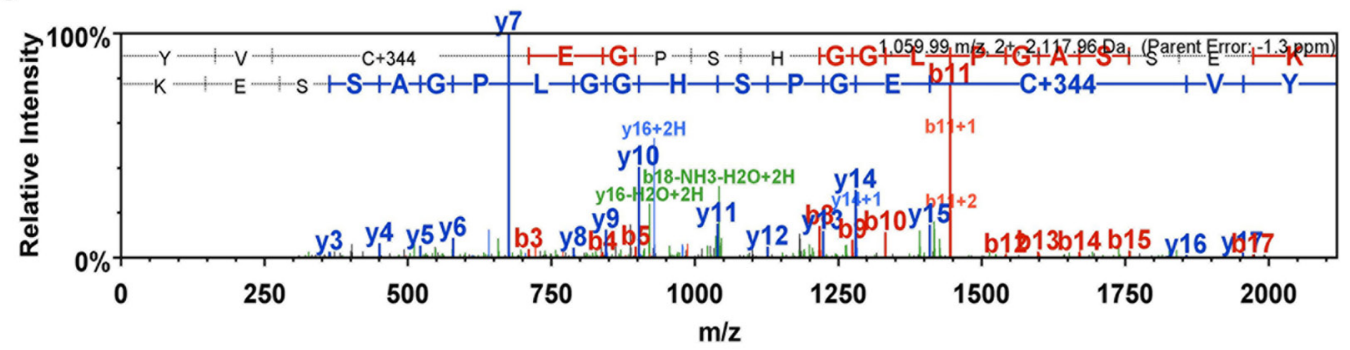

Figure 5: EriB directly targets the cysteine 62 of p50 through $\alpha, \beta$-unsaturated ketones. (A) SMMC-7721 cells were incubated with $1 \mu \mathrm{M} \mathrm{EBF7}$ (probe of reductive EriB) or EBF6, and the fluorescence was observed under microscopy. Nucleus and EBF6 were recognized by the red and blue fluorescence respectively. (B) Cytoplamic and nucleic fractions of SMMC-7721 cells lysates were treated with $1 \mu \mathrm{M}$ EBF7 or EBF6. After SDS-PAGE, the samples were exposed to UV light. (C) SMMC-7721 cells were transfected with $p 50^{W T}, p 50^{C 62 S}$ and $p 50^{C 1195}$, respectively, and treated with EBF6 $(3 \mu \mathrm{M})$ for $3 \mathrm{~h}$. The fluorescence was detected under microscope. The p50 antibody was used to detect the expression of the protein with Western blot assay. (D) The purified FLAG tagged human p50 and p50 variant proteins were incubated with $1 \mu \mathrm{M}$ EBF6 for $1 \mathrm{~h}$ respectively. After SDS-PAGE, the samples were exposed to UV light with p50 as the loading control. (E) Human recombinant p50 protein was incubated without or with EriB $(1 \mu \mathrm{M})$ and subjected to liquid chromatographymass spectra analysis. (F) The binding model of EriB in the DNA binding region (DBR) of p50. The yellow dotted lines represent hydrogen bonds between EriB (cyan) and the related residues of p50 (blue).

To investigate if Cys62 of p50 is the residue that EriB bound with, the mutants of $\mathrm{p} 5062 \mathrm{Cys} \rightarrow \operatorname{Ser}\left(p 50^{\mathrm{C} 62 \mathrm{~S}}\right)$ and p50 119Cys $\rightarrow \operatorname{Ser}\left(p 50^{C 119 S}\right)$ were constructed. SMMC7721 cells transfected with wild type $\mathrm{p} 50\left(p 50^{w t}\right), p 50^{\mathrm{C} 62 \mathrm{~S}}$ and $p 50^{C 62 S}$ plasmids were treated with EBF6 respectively, and were observed under the fluorescence microscope. Compared with the vector transfected group, the intensities of the fluorescence obviously increased in both $p 50^{w t}$ and $p 50^{\text {C119S }}$ transfected cells, while hardly changed in the p50 ${ }^{C 62 S}$ transfected cells (Figure 5c), suggesting the Cys62 is required for the binding of EriB with p50.

FLAG tagged proteins of p50wt and the mutant p50C62S and p50C119S were expressed in
SMMC- 7721 cells and purified, and then incubated with EBF6 respectively, followed by SDS-PAGE (Figure 5d). Compared with the wide type p50 and p50C119S, the interaction between EBF6 and p50C62S was abolished, which confirmed EriB bound to the cysteine 62 of p50. The covalent modification of cysteine 62 of p 50 by EriB was further confirmed by mass spectra analysis. As shown in Figure 5e, a 344 Da increase (the molecular weight of EriB) was found at the Cys62 of the peptide (YVCEGPSHGGLPGASSEK) of p50 (Figure 5e).

Besides, the binding model of EriB to p50 was demonstrated based on molecular docking. As shown in Figure 5f, the modeled EriB-p50 complex has the Michael 
acceptor at C-17 which could form a C-S bond with Cys62, and the following favorable interaction between EriB and p50 were also observed: a hydrogen bond between the C-7 hydroxyl group with the Glu63 carbonyl group and the hydrogen bonds between the C-6, C-7 hydroxyl groups and the Arg59 NH group.

\section{P50 knock-down attenuates the cytotoxicity and the apoptosis induced by EriB}

To investigate whether p50 is required for EriB to exert its anti-tumor activity, cell proliferation was studied in cells with p50 knocked down by small interfering RNA (siRNA). Luciferase assay showed that p50 knockdown compromised the NF- $\mathrm{\kappa B}$ transcriptional activity suppressed by EriB (Figure 6a). According to the growth curve analysis (Figure 6b), EriB showed obvious inhibitory effects on the proliferation of SMMC-7721 cells (difference between the red curve and the black line). As a result, siRNA mediated knock-down of p50 in SMMC-7721 cells eliminated the antiproliferative activity of EriB, deduced from the cell growth curves (the green curve and the blue curve in Figure $6 \mathrm{~b}$ ). The $\mathrm{IC}_{50}$ of EriB toward cells transfected with p50 siRNA was determined using MTT assay (Figure 6c), with scrambled siRNA as the control. As the result showed, transfection of p50 siRNA attenuated efficiently the cytotoxic activity of EriB, though not remarkable due to probably the relatively low efficiency of interference. Cell apoptosis assay revealed that the apoptotic effect of EriB was markedly weakened in SMMC-7721 cells with p50 knocked down by siRNA (Figure 6d). Furthermore, the intensity of the fluorescence of bound EriB to p50 decreased obviously in p 50 knockeddown SMMC-7721 cells, comparing with the control cells (Figure 6e). These data suggest that the interaction between EriB and p50 is required for the anti-tumor activity of EriB.

\section{DISCUSSION}

As an ent-kaurene diterpenoid isolated from Isodon eriocalyx var. Laxiflora, Eriocalyxin B (EriB) possesses potent bioactivity of antitumor and anti-autoimmune
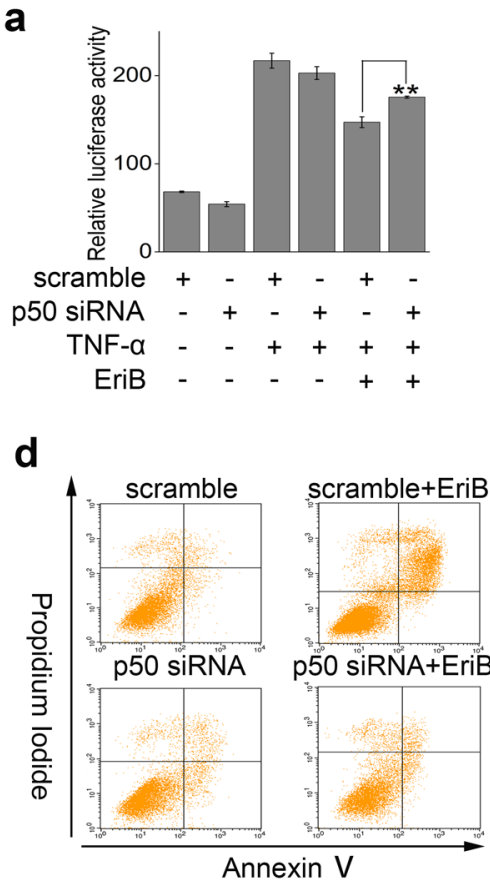

b
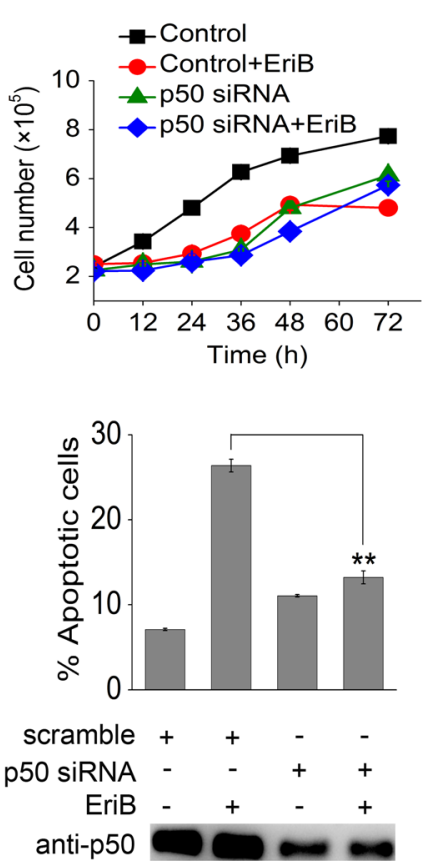

C

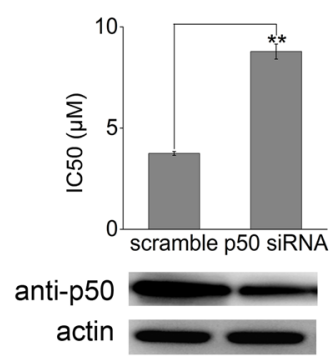

e

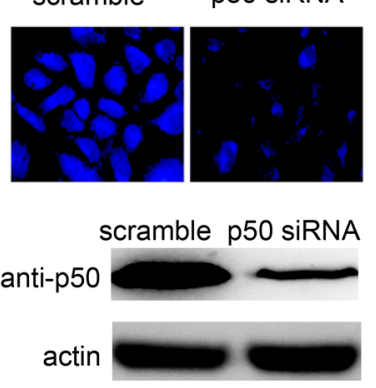

Figure 6: P50 knock-down attenuates the cytotoxicity and apoptosis inducing activity of EriB. (A) HEK 293T cells were transiently transfected with scrambled siRNA or p50 siRNA for $48 \mathrm{~h}$, then pretreated with $1 \mu \mathrm{M}$ EriB and stimulated with $25 \mathrm{ng} / \mathrm{mL}$ TNF- $\alpha$ for $18 \mathrm{~h}$. Cells were subjected to the analysis of NF-kB luciferase activity. The values represent the mean \pm S.D. ( $\mathrm{n}=3$ ). (B) The SMMC7721 cells were transfected with p50 small interfering RNA for $48 \mathrm{~h}$, and the cell count was documented at $0,12,24,36,48$ and $72 \mathrm{~h}$ in the absence or presence of $\operatorname{EriB}(1 \mu \mathrm{M})$, respectively. The values represent the mean \pm S.D. $(n=3)$. (C) Cytotoxic effects induced by EriB after $48 \mathrm{~h}$ treatment toward SMMC-7721 cells transfected with p50 siRNA were determined using MTT assays. The values represent the mean \pm S.D. (n=3). (D) SMMC-7721 cells were treated with EriB $(1 \mu \mathrm{M})$ for $48 \mathrm{~h}$ after p50 siRNA transfection. Apoptosis was analyzed by Annexin V-FITC/PI staining and the ratios of apoptotic cells were quantified. The corresponding p50 level was analyzed by Western blotting. The values represent the mean \pm S.D. $(n=3)$. (E) SMMC-7721 cells were transfected with p50 siRNA and treated with EBF6 (3 $\mu$ M) for $3 \mathrm{~h}$. The fluorescence was detected under microscope. The corresponding p50 level was analyzed by Western blotting. All the experiments were repeated three times. The values represent the mean \pm S.D. $(n=3)$. Statistical significance was analyzed by One-way ANOVA, $* * p<0.01$. 
inflammation and has received largely the attention from both chemistry and biology. Though extensive investigations of the mechanism involved in the inhibition of NF- $\kappa \mathrm{B}$ signaling by EriB have been reported, despite of some controversies, the direct targets of EriB remain elucidated, which became the aim of this resent study. Our findings showed that EriB-induced tumor cell apoptosis was associated with inactivation of NF- $\kappa \mathrm{B}$ signaling, which is consistent with previous studies [2-6]. We then synthesized the activity-based probes (ABPs) of EriB and with the favor of the ABBP technology, we identified p50 protein as the direct cellular target of EriB in SMMC-7721 cells.

$\mathrm{P} 50$ is one of the key regulators of NF- $\kappa \mathrm{B}$ signaling, which forms either homodimers or heterodimers and translocates into nucleus and activate the transcription of the target genes of the pathway upon the activation of the signaling. Either the dimerization or the cellular distribution of $\mathrm{p} 50 / \mathrm{p} 65$ in our current study turned out to be not affected by the binding of EriB with p50, which is consistent with previous studies $[4,5]$. It was also reported that EriB-mediated NF- $\kappa$ B inactivation of acute myeloid leukemia cells and the ovarian cancer stem cells was associated with the inhibition of nuclear translocation of $\mathrm{NF}-\kappa \mathrm{B}[2,3]$. We assumed that the difference could be due to the different types of cells tested and experimental conditions, such as the doses and time used for EriB treatment.

The direct interaction between EriB and Cys62 of p50 was confirmed in the present study by mass spectra analysis which showed that EriB directly interacted with the Cys62 of p50. Based on the kinetic assay with total nuclear lysates, it was showed that EriB works noncompetitively to inhibit NF- $\mathrm{KB}$ /DNA binding in previous studies [4]. Our data may suggest EriB inhibits the binding of p65/p50 complex to DNA through direct interaction with p50.

At the last part, the present study further showed that the interaction between EriB and p50 is crucial for the antitumor activity of EriB, which verified that functionally p50 is the cellular antitumor target of EriB. Downregulation of p50 using small interfering RNA attenuated the $\mathrm{NF}-\kappa \mathrm{B}$ transcriptional activity, antiproliferative activity, cytotoxic activity and apoptotic activity induced by EriB in SMMC-7721 cells, which might be due to the possibility that other NF- $\mathrm{B}$ family members, which EriB cannot interfere with, participate the signaling transduction and activate the NF- $\kappa$ B pathway [36].

In our present study, a couple of proteins appeared relatively weakly on the gel when pulled down with the dansyl EriB besides p50. Though we couldn't ruled out the possibility that EriB may also covalently bind with other proteins at the moment (Supplementary Information, Figure S3), we propose p50 is the major antitumor target of EriB based on our findings.

\section{CONCLUSION}

Activity-based protein profiling is a forwardchemical proteomic tool that uses specially designed chemical probes to fish mechanistically-related protein $[13,37]$. With the favor of the technology, we identified p50 protein as the direct target of EriB for the first time and illustrated the structural basis for their binding. The fact that EriB has already proved to be effective suppressing tumor growth both in vitro and in vivo highlights that p50 may be a very promising drug target for cancer therapy and EriB can be used as a natural chemical template for the development of p50 targeted therapeutic agents.

\section{MATERIALS AND METHODS}

\section{Isolation of EriB and synthesis of the ABPs}

Synthetic schemes, experimental procedures and characterization data of all new compounds are detailed in Supplementary Information.

\section{Cells and plasmids}

Hepatocellular carcinoma SMMC-7721 and HEK293T cells were obtained from Shanghai cell bank in China. All the cells were cultured in RPMI-1640 or DMEM medium (HyClone, Logan, UT), supplemented with 10\% fetal bovine serum (HyClone, Logan, UT), 100 units $/ \mathrm{mL}$ penicillin and $0.1 \mathrm{mg} / \mathrm{mL}$ streptomycin (HyClone) at $37{ }^{\circ} \mathrm{C}$ in a humidified atmosphere with $5 \% \mathrm{CO}_{2} . p 50^{w t}, p 50^{C 62 S}$ and $p 50^{C 119 S}$ were constructed by site-directed mutagenesis based on pCMV-p105 (Thermo Scientific, Rockford, IL).

\section{MTT assay}

Cytotoxicity of compounds was determined by MTT method. Briefly, cells were plated in 96-well plates $12 \mathrm{~h}$ before treatment and continuously exposed to test compounds for $48 \mathrm{~h}$. Then MTT (Sigma-Aldrich, St. Louis, MO) was added to each well. The samples were incubated at $37{ }^{\circ} \mathrm{C}$ for $4 \mathrm{~h}$ and formazan produced was dissolved by the addition of $200 \mu \mathrm{L} /$ well of Trevogen, followed by a further incubation at $37^{\circ} \mathrm{C}$ overnight in the dark. The optical density (OD) was measured at $595 \mathrm{~nm}$ using a microplate reader (Bio-Rad Laboratories). The $\mathrm{IC}_{50}$ values are calculated from appropriate dose-response curves.

\section{Cell apoptosis analysis}

Cell apoptosis was analyzed using the Annexin V-FITC/PI Apoptosis kit (BD Biosciences, Franklin Lakes, NJ) according to the manufacturer's protocols. Cells were 
seeded in 6-well plates at a density of $1.2 \times 10^{6}$ cells/ well. After indicated treatment cells were collected and then washed twice with cold PBS, and then resuspended in a binding buffer containing Annexin V-FITC and propidium iodine (PI). After incubation for 15 minutes at room temperature in the dark, the fluorescent intensity was measured using a FACSCalibur flow cytometer (BD Biosciences, Franklin Lakes, NJ).

\section{Western blotting}

Cells were harvested and lysed in SDS sample buffer (62.5 mM Tris-HCl, pH 6.8, 10\% glycerol, 2\% SDS, $50 \mathrm{mM}$ DTT and $0.01 \%$ bromphenol blue). Lysates were subjected to SDS-PAGE and transferred to PVDF membranes (Millipore). Membranes were blocked with $5 \%$ nonfat milk in Tris-buffered saline $/ 0.1 \%$ Tween-20 and incubated at $4{ }^{\circ} \mathrm{C}$ overnight with the following antibodies: anti-bcl-2 (Santa Cruz, CA), anti-bcl-xL (Santa Cruz, CA), anti-p65 (Santa Cruz, CA), anti-XIAP (Epitomics), anti-survivin (Santa Cruz, CA), anti-caspase 3 (Santa Cruz, CA), anti-cleaved caspase 3 (Santa Cruz, CA), anti-caspase 9 (Epitomics), anti-caspase 8 (Santa Cruz, CA), anti-PARP-1 (Santa Cruz, CA), anti-Lamin $\mathrm{A} / \mathrm{C}$ (Epitomics) and against $\beta$-actin (Santa Cruz, CA), followed by the corresponding horseradish peroxidaseconjugated secondary antibodies. Proteins of interest were visualized and imaged under chemi-luminescent detection using LASmini 4000 (GE Healthcare).

\section{Cell transfection and luciferase reporter assay}

HEK293T cells were transiently transfected with p65-Luc (Beyotime Institute for Biotechnology, China) and pRL-TK (Promega, Madison, WI) plasmids using Lipofectamine 2000 (Invitrogen, Carlsbad, CA) for $3 \mathrm{~h}$ in a 48-well plate. Cells were incubated with compounds for $1 \mathrm{~h}$ and subsequently activated with TNF- $\alpha$ (Peprotech, Rocky Hill, NJ). Luciferase activities were measured using the Dual-Luciferase Reporter Assay kit (Promega, Madison, WI).

\section{Nuclear and cytoplasmlic fractionation}

Collected cells were lysed in lysis buffer A (10 mM HEPES, $10 \mathrm{mM} \mathrm{KCl}, 1.5 \mathrm{mM} \mathrm{MgCl}, 0.5 \mathrm{mM}$ DTT, pH 7.9) with $0.4 \%$ Nonidet P-40 and EDTA-free protease inhibitor cocktail (Roche, Indianapolis, IN, USA) for 10 minutes on ice. After being microcentrifuged for 5 minutes at $500 \mathrm{~g}$, the supernatants were collected as cytoplasmic extracts. Pellets were resuspended in lysis buffer B (20 mM HEPES, pH 7.9, 420 mM NaCl, $0.5 \mathrm{mM}$ DTT, $0.2 \mathrm{mM}$ EDTA, and 25\% glycerol), and incubated for 30 minutes on ice. After being centrifuged at $12,000 \mathrm{~g}$ for 10 minutes, supernatants were collected.

\section{Pull-down assay}

Total cell extracts were lysed and precipitated with biotinyl-EriB for $2 \mathrm{~h}$ at room temperature, followed by addition of ImmunoPure immobilized streptavidin beads (Thermo Scientific, Rockford, IL) at $4{ }^{\circ} \mathrm{C}$ overnight. After washing for five times with the lysis buffer, the beads were boiled in the presence of the SDS sample buffer. The proteins were separated on $10 \%$ SDS-polyacrylamide gels and the specific binding proteins were detected with coomassie brilliant blue or p50 antibody (Epitomics, Burlingame, California).

\section{Co-immunoprecipitation assay}

Extracts of SMMC-7721 cells were incubated with anti-p65 antibody (Santa Cruz, CA) for $2 \mathrm{~h}$, and then incubated with protein $\mathrm{G}$ plus/protein A agarose (Santa Cruz, CA) at $4{ }^{\circ} \mathrm{C}$ overnight. Samples were washed five times with $50 \mathrm{mM}$ Tris-HCl, pH 7.9, $150 \mathrm{mM} \mathrm{NaCl}, 1 \%$ NP-40 and subjected to Western blotting analysis.

\section{Immunofluorescence staining}

Cells were grown on chamber slides for $18 \mathrm{~h}$ before treatment. Drug-treated cells were fixed in $4 \%$ paraformaldehyde for 20 minutes. After being washed in PBS, the slides were treated by $0.1 \%$ Triton-X, blocked with $3 \%$ BSA in PBS, incubated with anti-p65 antibody (Santa Cruz, CA) overnight, washed with PBS, and incubated with corresponding FITC conjugated secondary antibody (Sigma-Aldrich) for $1 \mathrm{~h}$. The slides were then incubated with DAPI (4',6-diamidino-2-phenylindole) for 10 minutes and observed under microscopy (Eclipse Ti, Nikon).

\section{Chromatin immunoprecipitation assay}

ChIP was performed in SMMC-7721 cells using SimpleChIP ${ }^{\circledR}$ Enzymatic Chromatin IP Kit (Cell Signaling Technology, Trask Lane Danvers, MA, USA) according to the manufacturer's instructions. The purified DNA was subjected to PCR amplification using the human Bcl-xL promoter specific primers [38]. forward 5'-GCACCACCTACATTCAAATCC-3' and reverse 5'-CGATGGAGGAGGAAGCAAGC-3'. PCR fragments were analyzed on agarose gel and the size (251 bp) was compared to a molecular weight marker.

\section{Expression of $\mathrm{p50}^{\mathrm{wt}}, \mathrm{p50}^{\mathrm{C} 62 \mathrm{~S}}$ and $\mathrm{p50}^{\mathrm{C} 119 \mathrm{~S}}$ proteins}

SMMC-7721 cells were transfected with FLAG-p50 and C62S, C119S mutants for $24 \mathrm{~h}$, and the proteins were purified by ANTI-FLAG ${ }^{\circledR}$ M2 Affinity Gel (SigmaAldrich) according to the manufacturer's instructions. The purified proteins were eluted with $3 \times$ FLAG peptide (Sigma-Aldrich) and stored at $-20^{\circ} \mathrm{C}$ for use. 


\section{Liquid chromatography-mass spectra analysis}

The detailed protocols were described in Supporting Information.

\section{Molecular modeling of EriB with p50}

EriB was prepared (generating stereoisomers and valid single 3D conformers) by means of the Ligand Preparation module in Discovery Studio 3.5 (Accelrys). The crystal structure of p50 was retrieved from the Protein Data Bank (PDB entry: 3GUT). All crystallographic water molecules and DNA were removed from the coordinate set. Gold 4.0 (CCDC) was used for docking. The DNA binding domain, amino acids 59-71, was defined as the binding pocket on the basis of the previous studies[31-34]. In the docking process, the standard docking score was used to rank the docking conformations. All the parameters were set as the default values, except that a maximum number of 300,000 operations and a population of 200 individuals were imposed. The GoldScore fitness function and ChemScore fitness function were used sequentially, and the best-fitting binding mode was identified.

\section{Small interfering RNAs}

Duplex siRNAs with two thymidine residues $(\mathrm{dTdT})$ at the 3'-end of sequence were synthesized at GenePharma (Shanghai, China). The target sequences were as follows [38]:

p50: 5'-GUCACUCUAACGUAUGCAA-3'.

Scrambled control: 5'-UUCUCCGAACGUGUCA CGU-3'

SiRNAs were transfected into SMMC-7721 cells using Lipofectamine 2000.

\section{Conflict of interest}

The authors declare no conflict of interest.

\section{ACKNOWLEDGEMENTS}

This work was supported by the National Natural Science Fundation of China (No. 90813004, 21302193, U0932602), the 100 Talents Program of the Chinese Academy of Sciences (Y. Li), the Major State Basic Research Development Program of China (2011CB915503), and the projects of Sciences and Technology of Yunnan Province (2009CI120, 2013FA047). We thank Dr. Hongyu Zhou for critical reading of the manuscript.

\section{REFERENECES}

1. Sun HD, Huang SX, Han QB. Diterpenoids from Isodon species and their biological activities. Natural Product Reports. 2006; 23:673-698.
2. Wang L, Zhao WL, Yan JS, Liu P, Sun HP, Zhou GB, Weng ZY, Wu WL, Weng XQ, Sun XJ, Chen Z, Sun HD, Chen SJ. Eriocalyxin B induces apoptosis of $t(8 ; 21)$ leukemia cells through NF-kappaB and MAPK signaling pathways and triggers degradation of AML1-ETO oncoprotein in a caspase-3-dependent manner. Cell Death Differ. 2007; 14:306-317.

3. Leizer AL, Alvero AB, Fu HH, Holmberg JC, Cheng YC, Silasi DA, Rutherford T, Mor G. Regulation of inflammation by the NF-kappaB pathway in ovarian cancer stem cells. Am J Reprod Immunol. 2011; 65:438-447.

4. Leung CH, Grill SP, Lam W, Gao W, Sun HD, Cheng YC. Eriocalyxin B inhibits nuclear factor-kappaB activation by interfering with the binding of both $\mathrm{p} 65$ and p50 to the response element in a noncompetitive manner. Mol Pharmacol. 2006; 70:1946-1955.

5. Zhang YW, Jiang XX, Chen QS, Shi WY, Wang L, Sun HD, Shen ZX, Chen Z, Chen SJ, Zhao WL. Eriocalyxin B induces apoptosis in lymphoma cells through multiple cellular signaling pathways. Exp Hematol. 2010; 38:191-201.

6. Lu Y, Chen B, Song JH, Zhen T, Wang BY, Li X, Liu P, Yang X, Zhang QL, Xi XD, Chen SD, Zuo JP, Chen Z, et al. Eriocalyxin B ameliorates experimental autoimmune encephalomyelitis by suppressing Th1 and Th17 cells. Proc Natl Acad Sci U S A. 2013; 110:2258-2263.

7. Li L, Yue GG, Lau CB, Sun H, Fung KP, Leung PC, Han Q, Leung PS. Eriocalyxin B induces apoptosis and cell cycle arrest in pancreatic adenocarcinoma cells through caspaseand p53-dependent pathways. Toxicol Appl Pharmacol. 2012; 262:80-90.

8. Cichon MA, Radisky DC. ROS-induced epithelialmesenchymal transition in mammary epithelial cells is mediated by NF-kB-dependent activation of Snail. Oncotarget. 2014; 5:2827-2838.

9. Siveen KS, Mustafa N, Li F, Kannaiyan R, Ahn KS, Kumar AP, Chng WJ, Sethi G. Thymoquinone overcomes chemoresistance and enhances the anticancer effects of bortezomib through abrogation of NF-kappaB regulated gene products in multiple myeloma xenograft mouse model. Oncotarget. 2014; 5:634-648.

10. Dalmases A, Gonzalez I, Menendez S, Arpi O, Corominas JM, Servitja S, Tusquets I, Chamizo C, Rincon R, Espinosa L, Bigas A, Eroles P, Furriol J, et al. Deficiency in p53 is required for doxorubicin induced transcriptional activation of NF-small ka, CyrillicB target genes in human breast cancer. Oncotarget. 2014; 5:196-210.

11. Huang H, Ma L, Li J, Yu Y, Zhang D, Wei J, Jin H, Xu D, Gao J, Huang C. NF-kappaB1 inhibits c-Myc protein degradation through suppression of FBW7 expression. Oncotarget. 2014; 5:493-505.

12. Agarwal NK, Zhu X, Gagea M, White CL 3rd, Cote G, Georgescu MM. PHLPP2 suppresses the NF-kappaB pathway by inactivating IKKbeta kinase. Oncotarget. 2014; $5: 815-823$ 
13. Cravatt BF, Wright AT, Kozarich JW. Activity-based protein profiling: from enzyme chemistry to proteomic chemistry. Annu Rev Biochem. 2008; 77:383-414.

14. Peng SC, Wong DS, Tung KC, Chen YY, Chao CC, Peng $\mathrm{CH}$, Chuang YJ, Tang CY. Computational modeling with forward and reverse engineering links signaling network and genomic regulatory responses: NFkappaB signaling-induced gene expression responses in inflammation. BMC Bioinformatics. 2010; 11:308.

15. Kucharczak J, Simmons MJ, Fan Y, Gelinas C. To be, or not to be: NF-kappaB is the answer-role of Rel/NFkappaB in the regulation of apoptosis. Oncogene. 2003; 22:8961-8982.

16. Bavi P, Uddin S, Bu R, Ahmed M, Abubaker J, Balde V, Qadri Z, Ajarim D, Al-Dayel F, Hussain AR, Al-Kuraya KS. The biological and clinical impact of inhibition of NF-kappaB-initiated apoptosis in diffuse large B cell lymphoma (DLBCL). J Pathol. 2011; 224:355-366.

17. Mita AC, Mita MM, Nawrocki ST, Giles FJ. Survivin: key regulator of mitosis and apoptosis and novel target for cancer therapeutics. Clin Cancer Res. 2008; 14:5000-5005.

18. Fiandalo MV, Kyprianou N. Caspase control: protagonists of cancer cell apoptosis. Exp Oncol. 2012; 34:165-175.

19. Chaitanya GV, Steven AJ, Babu PP. PARP-1 cleavage fragments: signatures of cell-death proteases in neurodegeneration. Cell Commun Signal. 2010; 8:31.

20. Salvesen GS, Duckett CS. IAP proteins: blocking the road to death's door. Nat Rev Mol Cell Biol. 2002; 3:401-410.

21. Zhao Y, Niu XM, Qian LP, Liu ZY, Zhao QS, Sun HD. Synthesis and cytotoxicity of some new eriocalyxin B derivatives. Eur J Med Chem. 2007; 42:494-502.

22. Liu Y, Lok C-N, Ko BC-B, Shum TY-T, Wong M-K, Che C-M. Subcellular Localization of a Fluorescent Artemisinin Derivative to Endoplasmic Reticulum. Org Lett. 2010; 12:1420-1423.

23. Pereira SG, Oakley F. Nuclear factor-kappaB1: regulation and function. Int J Biochem Cell Biol. 2008; 40:1425-1430.

24. Ghosh G, Wang VY, Huang DB, Fusco A. NF-kappaB regulation: lessons from structures. Immunol Rev. 2012; 246:36-58.

25. Mellits KH, Hay RT, Goodbourn S. Proteolytic degradation of MAD3 (I kappa B alpha) and enhanced processing of the NF-kappa B precursor p105 are obligatory steps in the activation of NF-kappa B. Nucleic Acids Res. 1993; 21:5059-5066.

26. Sen N, Paul BD, Gadalla MM, Mustafa AK, Sen T, Xu R, Kim S, Snyder SH. Hydrogen sulfide-linked sulfhydration of NF-kappaB mediates its antiapoptotic actions. Mol Cell. 2012; 45:13-24.

27. Atsmon J, Sweetman BJ, Baertschi SW, Harris TM, Roberts LJ 2nd. Formation of thiol conjugates of 9-deoxy-delta 9,delta 12(E)-prostaglandin D2 and delta 12(E)-prostaglandin D2. Biochemistry. 1990; 29:3760-3765.

28. Gersch M, Kreuzer J, Sieber SA. Electrophilic natural products and their biological targets. Nat Prod Rep. 2012; 29:659-682.

29. Metelev V, Romanenkov A, Kubareva E, Zubin E, Polouchine N, Zatsepin T, Molochkov N, Oretskaya T. Structure-based cross-linking of NF-kappaB p50 homodimer and decoy bearing a novel 2'-disulfide trapping site. IUBMB Life. 2006; 58:654-658.

30. Nishi T, Shimizu N, Hiramoto M, Sato I, Yamaguchi Y, Hasegawa M, Aizawa S, Tanaka H, Kataoka K, Watanabe H, Handa H. Spatial redox regulation of a critical cysteine residue of NF-kappa B in vivo. J Biol Chem. 2002; 277:44548-44556.

31. Muller CW, Rey FA, Sodeoka M, Verdine GL, Harrison SC. Structure of the NF-kappa B p50 homodimer bound to DNA. Nature. 1995; 373:311-317.

32. Berkowitz B, Huang DB, Chen-Park FE, Sigler PB, Ghosh G. The x-ray crystal structure of the NF-kappa B p50.p65 heterodimer bound to the interferon beta -kappa B site. J Biol Chem. 2002; 277:24694-24700.

33. Prasad AS, Bao B, Beck FW, Sarkar FH. Zinc activates NF-kappaB in HUT-78 cells. J Lab Clin Med. 2001; 138:250-256.

34. Chen FE, Huang DB, Chen YQ, Ghosh G. Crystal structure of p50/p65 heterodimer of transcription factor NF-kappaB bound to DNA. Nature. 1998; 391:410-413.

35. Matthews JR, Kaszubska W, Turcatti G, Wells TN, Hay RT. Role of cysteine62 in DNA recognition by the P50 subunit of NF-kappa B. Nucleic Acids Res. 1993; 21:1727-1734.

36. Hoffmann A, Leung TH, Baltimore D. Genetic analysis of NF-kappaB/Rel transcription factors defines functional specificities. EMBO J. 2003; 22:5530-5539.

37. Berger AB, Vitorino PM, Bogyo M. Activity-based protein profiling: applications to biomarker discovery, in vivo imaging and drug discovery. Am J Pharmacogenomics. 2004; 4:371-381.

38. Schmitt AM, Crawley CD, Kang S, Raleigh DR, Yu X, Wahlstrom JS, Voce DJ, Darga TE, Weichselbaum RR, Yamini B. p50 (NF-kappaB1) is an effector protein in the cytotoxic response to DNA methylation damage. Mol Cell. 2011; 44:785-796. 